\title{
Moon illusion simulated in complete darkness: Planetarium experiment reexamined
}

\author{
KOTARO SUZUKI \\ Niigata University, Niigata, Japan
}

\begin{abstract}
In 1962, Kaufman and Rock reported that the moon illusion did not occur in the darkness of a planetarium or in a completely dark room. The present study reexamined their findings. Two pairs of light points, separated by $3.5^{\circ}$, were presented on the dome screen of a planetarium. Subjects compared the distance between the two light points presented in the horizontal direction with the distance between the two light points at the zenith. Three illumination conditions were used: The inside of the planetarium was completely dark, was lighted, or was projected with the silhouette of a city under a starry sky. The effect of eye elevation on the illusion was also examined. Contrary to Kaufman and Rock's results, a size discrepancy comparable to the moon illusion was obtained in the horizon-and-stars condition and even in the complete-darkness condition. Little or no illusion was obtained in the lighted-room condition. The results also showed that eye elevation affected the magnitude of the illusion.
\end{abstract}

In 1962, after conducting one experiment at the Hayden Planetarium in New York in August 1956, Kaufman and Rock reported that the moon illusion does not exist in complete darkness. In complete darkness, they projected a light disk onto the horizon screen and another light disk onto the zenith screen. These disks were equidistant $(37.5 \mathrm{ft}$, or $11.4 \mathrm{~m})$ from subjects who were asked to compare the sizes of the two disks. The horizon disk appeared to be 1.03 times larger than the zenith disk; that is, there was little illusion. They then conducted a further experiment in a completely dark room, using an apparatus that could present light disks of optically infinite distance. The subjects were presented with one disk in the horizontal direction and another at the zenith. The horizon disk again appeared to be only 1.03 times larger than the zenith disk. From the results of these two experiments, they concluded that the moon illusion does not exist in complete darkness. This conclusion provided them with a basis on which they conducted further experiments in an outdoor setting and developed their terrain hypothesis for the moon illusion (Kaufman \& Rock, 1962; Rock \& Kaufman, 1962).

More than 30 years before Kaufman and Rock's reports, however, Schur (1925) conducted similar experiments in large rooms (e.g., an airplane hangar and a church) and did obtain the moon illusion: the horizon disk appeared to be larger than the zenith disk. The obtained size ratios of the horizon disk to the zenith disk became larger with

\footnotetext{
Thanks are extended to Masakuni Goto, Yukikazu Hine, and the astronomical section of the Niigata Science Museum for use of the planetarium, and to Hidehito Maeyama for assistance with the experiment, and to Hitoshi Honda for helpful suggestions on the experiment. Requests for reprints should be sent to Kotaro Suzuki, Department of Psychology, Faculty of Humanities, Niigata University, Ikarashi, Niigata 95021, Japan.
}

viewing distance: $1.13,1.16,1.21,1.34,1.42$, and 1.50 at $3,4.8,6,13.5,22$, and $33 \mathrm{~m}$, respectively. Leibowitz and Hartman (1959) also reported the illusion obtained in darkness in a theater. At a viewing distance of $35 \mathrm{ft}$ $(10.7 \mathrm{~m})$, the horizon disk appeared to be 1.2 to 1.3 times larger than the zenith disk. Thus, despite the use of similar apparatus and situations, Kaufman and Rock's results were the opposite of Schur's and Leibowitz and Hartman's. Kaufman and Rock suspected that Schur's and Leibowitz and Hartman's experiments were not done in complete darkness. They suggested that some stray light from the projectors might have made the room visible, and that the illusion might occur because of the visibility of cues in the room that would affect size and distance perception. That is, they interpreted Schur's and Leibowitz and Hartman's results in terms of visible objects in the terrain (Rock \& Kaufman, 1962; Kaufman \& Rock, 1989).

In the present study, I reexamined Kaufman and Rock's experiments. With regard to their planetarium experiment, there are some problems: (1) Although they reported that the planetarium was completely dark, it might not always have been so. The light disks projected on the screen subtended $1.5^{\circ}$ in visual angle, and reflected light from these large-size disks might have made objects in the planetarium visible. Therefore, their experiment may not have been free of stray light, the problem they suggested concerning Schur's and Leibowitz and Hartman's experiments. (2) Kaufman and Rock did not show that the illusion could be obtained when stray light made objects in the planetarium visible. Thus, their interpretation of Schur's and Leibowitz and Hartman's results is unpersuasive. (3) The number of subjects participating in Kaufman and Rock's planetarium experiment was small; they used only 5 subjects. A small number of subjects were also used in Holway and Boring's (1940a, 1940b) and Schur's (1925) experiments, and this has occasionally 
been suggested as a source of unreliability in the data. This concern could be applied to Kaufman and Rock's planetarium experiment. (4) Finally, with regard to Kaufman and Rock's dark-room experiment, the optically infinite distance of the stimuli, on which they insisted, might not be useful in complete darkness, although it has been quite important in outdoor-setting experiments.

To deal with these problems, the present study examined the questions of whether or not the moon illusion exists in complete darkness and whether or not it occurs in a planetarium. The purposes and characteristics of the present study can be summarized as follows:

1. The standard and comparison stimuli were pairs of small light points projected onto the screen of the planetarium. Because they were produced by laser beams, which enlarge little with their progression, the images of the light points formed on the screen were small and clear. Unlike the light disks employed in Kaufman and Rock's experiment, these light points on the screen had little reflection, and so did not make objects in the planetarium visible and permitted the planetarium to be completely dark. The subjects' task was to estimate the distance between two light points. That is, subjects were asked to adjust the distance between the two light points seen in the horizontal direction (comparison stimulus) to match the distance between two light points seen overhead (standard stimulus). With Stroobant's (1884) finding in mind-that the spacing between stars is relevant as a measure of the moon illusion-I decided it would be possible to use the starlike light points as stimuli instead of the moonlike light disks.

2 . In the present experiment, three illumination conditions were used: complete darkness, lighted room, and horizon and stars.

In the complete-darkness condition, subjects were led into the planetarium in complete darkness. In the experiment, they saw only the light points, and had no way of knowing exactly how things in the planetarium, such as seats, the screen, and the experimental apparatus, were arranged and how far away the light points actually were.

In the lighted-room condition, the inside of the planetarium was illuminated, and the subjects were able to see many objects in the planetarium, especially the objects interposed to the horizon screen. This condition was included to determine the possible effect of stray light as well as whether or not the illusion exists when the layout in the planetarium is visible.

In the horizon-and-stars condition, stars and the silhouette of a city on the horizon were projected onto the screen of the planetarium. In this condition, the setting approximated a natural, or outdoor, one, and it was expected that the magnitude of the obtained moon illusion would be close to that obtained in an outdoor setting.

3 . The present study also examined the effects of eye elevation on the illusion. Two eye-and-head-position conditions were introduced. In one condition, subjects were asked to keep their heads upright and to elevate their eyes while viewing the standard stimulus overhead. In the sec- ond condition, the subjects were asked to bend their necks and the upper part of their bodies backward in order to look straight ahead at the stimulus overhead. The effect of eye elevation on the illusion has been reported by several researchers (Hermans, 1954; Holway \& Boring, 1940a, 1940b; Schur, 1925; Zoth, 1899), but the effect was not obtained in Kaufman and Rock's experiments (Kaufman \& Rock, 1962; Rock \& Kaufman, 1962). The present study examines this discrepancy.

\section{METHOD}

\section{Subjects}

The subjects were 16 undergraduate students. Ten of them had uncorrected visual acuity of greater than 0.8 . The remaining 6 usually wore contact lenses and, with them, had a visual acuity of greater than 1.0. Students who wore spectacles were not used.

\section{Planetarium and Stimuli}

The experiment was conducted at the planetarium of the Niigata Science Museum. As shown in Figure 1, the dome of the planetarium was hemispheric in shape, $18.4 \mathrm{~m}$ in diameter in the central horizontal plane and $11.3 \mathrm{~m}$ high from the floor. Any position on the screen was equidistant $(9.2 \mathrm{~m})$ from the center of the planetarium. A projection apparatus for producing star images was located on a center platform, $4.8 \mathrm{~m}$ in diameter and $1 \mathrm{~m}$ in height. It was surrounded by a $1.5-\mathrm{m}$-wide circular path and then by seats for an audience.

Two pairs of light points (standard and comparison stimuli) were projected onto the dome screen in the horizontal and zenithal directions by two pairs of lasers. Both pairs of lasers were set equidistant from the center of the planetarium, those for the standard stimulus on the right side of the platform and those for the comparison stimulus on the left side. The projection distances of both stimuli were the same $(9.65 \mathrm{~m})$.

The standard stimulus was projected onto the uppermost position of the dome by two lasers placed at a distance of $2.95 \mathrm{~m}$ from the center of the planetarium. The heads of the two lasers were about $2.1 \mathrm{~m}$ high, that is, at the level of the central horizontal plane of the planetarium. To the subject, these overhead light points were located $72^{\circ}$ upward and appeared side by side. The distance between these two light points was $62 \mathrm{~cm}$ on the screen, subtending $3.5^{\circ}$ in visual angle to the subject's eyes.

The comparison stimulus was projected onto a position $90 \mathrm{~cm}$ above the central horizontal plane, that is, $3 \mathrm{~m}$ above the floor, by two lasers. This position was $3^{\circ}$ above the subject's eye level and was to be above the skyline in the projected scenery in the horizon-and-stars condition. A pair of lasers for the comparison stimulus were placed at the level of the central horizontal plane, $2.95 \mathrm{~m}$ away from the center of the planetarium. The subject was seated on a stool between these lasers. The position of the laser on the left side of the subject was fixed, whereas the laser on the right side could be moved laterally. So the subject could adjust the position of the light point on the right. The subject's eye level was about $2.1 \mathrm{~m}$, that is, the level of the central horizontal plane. The distance from the subject's eyes to the standard and comparison stimuli on the screen was $9.65 \mathrm{~m}$.

The lasers used were four HeNe lasers (NeoArk Neo-1M), which emitted red beams $(632.8 \mathrm{~nm})$. Except for their heads, the lasers were tightly sealed.

\section{Procedure}

The experiment was conducted by two experimenters. A blindfolded subject was led into the planetarium in complete darkness 

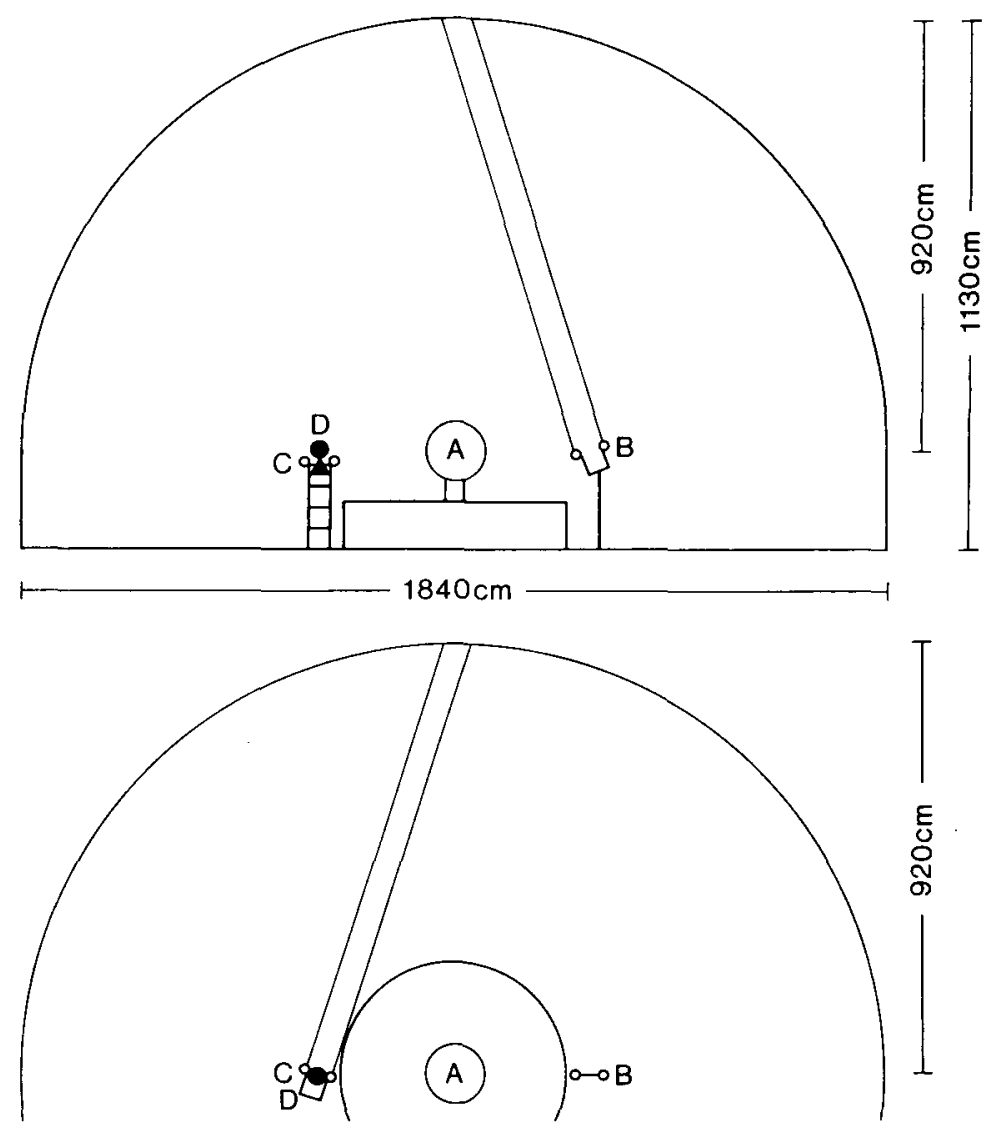

Figure 1. Layout of the planetarium: Side view (top) and top view (bottom). (A) Projection apparatus for stars on the center platform; (B) lasers for standard stimulus; (C) lasers for comparison stimulus; and (D) subject.

by one of the experimenters. After being seated on the stool, the subject was asked to adjust the position of the right-hand point of the comparison stimulus in the horizontal direction to equalize the distance between that pair of points and the distance between the two standard-stimulus points presented overhead. After every adjustment, the subject was required to close his or her eyes while the other experimenter measured and recorded the distance between the two light points in the horizontal direction.

\section{Conditions}

The following three illumination conditions and two eye-headposition conditions were used.

Complete-darkness condition. In the planetarium, any light rays from the outside were shut out. All illuminations, including emergency exit signs and small guide lamps, were turned off. Only the stimulus light points on the screen were seen in the complete darkness.

Lighted-room condition. Lights on the wall below the dome screen and around the star-projection apparatus on the center platform were on, making the layout in the planetarium-that is, the seats, the dome screen, and the star-projection apparatus-visible to the subject.

Horizon-and-stars condition. In darkness, a panoramic $\left(360^{\circ}\right)$, starry night scene of Niigata City, including a skyline, was presented on the screen. The skyline in the scene was $60 \mathrm{~cm}$ above the cen- tral horizontal plane, that is, $2.7 \mathrm{~m}$ above floor level. The scene of the city was projected from 12 slide projectors arranged on the center platform. Stars (only fixed stars) were projected on the screen by the star-projection apparatus on the platform.

Each subject served in two eye-position conditions for each of these three illumination conditions.

Eyes-elevated condition. The subject was instructed to maintain his/her head in the upright position while elevating his/her eyes to view the light points overhead. (Strictly speaking, it was impossible to elevate only the eyes to $72^{\circ}$ upward. Although elevating their eyes as much as possible [perhaps $60^{\circ}$ ], the subjects more or less bent their necks and/or the upper part of their bodies as necessary.)

Eyes-level condition. The subject was instructed to bend his/her neck and the upper portion of his/her body backward to view the light points overhead.

The complete-darkness condition was consistently executed first, before the subject knew the layout in the planetarium. For their second illumination condition, half of the subjects were assigned randomly, first to the lighted-room condition and then to the horizonand-stars condition. The other half served in the reverse order of conditions. For each illumination condition, presentation order of the two eye-head-position conditions was counterbalanced between the subjects.

The subjects performed in four trials, two ascending and two descending series, for each condition. 


\section{RESULTS}

The adjusted smaller (or larger) distance between the horizon light points than between the zenith light points means that the horizon stimulus was perceived as larger (or smaller) than the actually same-sized zenith stimulus. Thus, the ratios of the spacing (size) of the zenith standard stimulus to that of the horizon comparison stimulus were taken as indicating the magnitude of the illusion.

Table 1 shows the average ratios for each subject and their means. In the complete-darkness condition, the ratios were approximately 1.3. In the horizon-and-stars condition, ratios of approximately 1.2 were obtained. Onetailed $t$ tests for the single mean showed that the occurrence of the illusion was significant in these two illumination conditions $[t(15)=6.43$ for the eyes-elevated condition and $t(15)=5.69$ for the eyes-level condition in the complete-darkness condition, and $t(15)=4.93$ for the eyes-elevated condition and $t(15)=4.13$ for the eyeslevel condition in the horizon-and-stars condition; $p<$ .001 for each]. In the lighted-room condition, however, the ratios were less than 1.1 and the illusion seemed to be absent $[t(15)=1.51, .10<p<.05$, for the eyeselevated condition and $t(15)=.24$, n.s., for the eyeslevel condition, respectively].

The first condition for all subjects was complete darkness; the second and third conditions differed among the subjects. Preceding the analyses, the possible effect of presentation order was examined by comparing the results of the second and third conditions. The presentation order of the conditions had little effect on the results $[F(1,15)=$ .29 , n.s.].

A two-way analysis of variance was performed on the illumination and eye-head-position conditions. The effect of illumination was significant $[F(2,30)=24.03, p<$ $.001]$. The effect of the eye-head position was also sig-

Table 1

Average Size Ratios for the Illumination and Eye-Position Conditions

\begin{tabular}{|c|c|c|c|c|c|c|}
\hline \multirow[b]{2}{*}{ Subject } & \multicolumn{2}{|c|}{ Complete Darkness } & \multicolumn{2}{|c|}{ Lighted Room } & \multicolumn{2}{|c|}{ Horizon and Stars } \\
\hline & $\begin{array}{c}\text { Eyes } \\
\text { Elevated }\end{array}$ & $\begin{array}{l}\text { Eyes } \\
\text { Level }\end{array}$ & $\begin{array}{c}\text { Eyes } \\
\text { Elevated }\end{array}$ & $\begin{array}{l}\text { Eyes } \\
\text { Level }\end{array}$ & $\begin{array}{c}\text { Eyes } \\
\text { Elevated }\end{array}$ & $\begin{array}{l}\text { Eyes } \\
\text { Level }\end{array}$ \\
\hline 1 & 1.42 & 1.08 & .85 & .80 & 1.11 & .98 \\
\hline 2 & 1.03 & .95 & 1.01 & 1.06 & 1.27 & 1.08 \\
\hline 3 & 1.22 & 1.19 & 1.18 & 1.21 & 1.15 & 1.10 \\
\hline 4 & 1.24 & 1.52 & .91 & .85 & 1.22 & 1.13 \\
\hline 5 & 1.33 & 1.36 & 1.20 & 1.16 & 1.34 & 1.16 \\
\hline 6 & 1.48 & 1.28 & 1.43 & 1.28 & 1.71 & 1.48 \\
\hline 7 & 1.35 & 1.27 & 1.10 & 1.02 & 1.26 & 1.15 \\
\hline 8 & 1.25 & 1.25 & .74 & .69 & .83 & .90 \\
\hline 9 & 1.12 & 1.19 & 1.09 & 1.02 & 1.12 & 1.07 \\
\hline 10 & 1.84 & 1.28 & 1.33 & 1.03 & 1.60 & 1.40 \\
\hline 11 & 1.06 & 1.02 & .88 & .89 & .96 & .99 \\
\hline 12 & 1.59 & 1.57 & 1.18 & 1.09 & 1.59 & 1.31 \\
\hline 13 & 1.52 & 1.56 & 1.35 & 1.13 & 1.39 & 1.33 \\
\hline 14 & 1.39 & 1.34 & 1.15 & .97 & 1.36 & 1.17 \\
\hline 15 & 1.16 & 1.02 & .80 & .72 & 1.31 & 1.06 \\
\hline 16 & 1.24 & 1.22 & 1.00 & 1.10 & 1.18 & 1.20 \\
\hline$M$ & 1.33 & 1.26 & 1.08 & 1.00 & 1.28 & 1.16 \\
\hline$S D$ & .20 & .18 & .20 & .17 & .22 & .15 \\
\hline
\end{tabular}

nificant $[F(1,15)=12.33, p<.01]$. There was no interaction between these conditions $[F(2,30)=.81$, n.s. $]$. Further analyses showed that the differences between the complete-darkness and lighted-room conditions and between the horizon-and-stars and lighted-room conditions were significant $[F(1,15)=32.60, p<.001 ; F(1,15)$ $=35.88, p<.001$, respectively]. The difference between the complete-darkness and horizon-and-stars conditions was barely significant $[F(1,15)=4.15, .10<$ $p<.05]$. Eye elevation had a significant effect on the ratios in both the lighted-room and horizon-and-stars conditions $[F(1,15)=8.38, p<.05$, and $F(1,15)=20.04$, $p<.001$, respectively] but not in the complete-darkness condition $[F(1,15)=2.38$, n.s. $]$.

\section{DISCUSSION}

The main findings of the present experiment were the following: (1) A size discrepancy comparable to the moon illusion existed even in complete darkness, (2) a negligible illusion was obtained in the lighted room, and (3) eye elevation affected the magnitude of the illusion. These findings will be discussed below.

The moon illusion was obtained in the complete-darkness condition; the distance between the light points presented in the horizontal direction appeared to be about 1.3 times larger than that at the zenith. The result agrees with Leibowitz and Hartman's (1959) and Schur's (1925) findings. Thus, the illusion existed even in the absence of visual (retinal) cues for size and distance perception, such as a terrain scenery and the visible horizon.

What induced the illusion in complete darkness? The answer may lie in the availability of information (cues). In complete darkness, visual (retinal) cues to size and distance perception were absent, but vestibular and oculomotor cues are assumed to have been available, and such cues might play some role in obtaining the illusion in complete darkness. Thor and Wood suggested that the moon illusion depends on vestibular sensation (Thor \& Wood, 1966). Their hypothesis may account for the illusion obtained here, but they do not show strong evidence for the involvement of such information in the moon illusion. As for oculomotor cues, several researchers have recently proposed oculomotor hypotheses for the moon illusion. On the basis of results obtained in a series of experiments, Enright (1989a) claimed that the information provided by the oculomotor system, such as vergence, accommodation, and pupil size, induces the illusion. Iavecchia, Iavecchia, and Roscoe (1983) also showed that the state of accommodation of the eyes correlated with the apparent size of the moon. They suggested that the state of accommodation plays some role in the moon illusion. Leibowitz and Owens (1989) also suggested that the resting position of vergence relates to the moon illusion. Therefore, we can suppose that the illusion obtained in complete darkness was induced by the resting state of the oculomotor system, which might differ depending on viewing direction. Heuer and Owens (1989) showed that the resting position 
of vergence in darkness differed depending on the direction of the gaze: the resting position of vergence was closer with horizontal gaze than with elevated gaze. How this difference of the resting position of vergence produces the moon illusion was explained by Leibowitz and Owens (1989) in the following way. When distant objects, such as the moon, are viewed, the divergence effort is correspondingly greater in the horizontal direction than in the overhead direction. This effort provides a cue for size perception, so the horizon moon appears larger. Heuer and Owens's experimental setting, complete darkness, is quite similar to the complete-darkness condition in the present study. Thus, the illusion obtained in complete darkness can be explained in terms of oculomotor cues, especially the resting position of vergence.

In the lighted-room condition, unexpectedly, either no illusion was obtained or the illusion obtained was very small. Because many visual cues to size and distance perception were available and size and distance perception might be more correct, the illusion might be prevented or weakened. In this situation, some oculomotor cues were present, but they might not be used because of the dominance of the visual cues. Thus, it is not always true that the illusion occurs whenever there are many more cues to size and distance perception.

In the horizon-and-stars condition, the illusion did occur. The average ratio was 1.2 . The magnitude of the illusion was the same as or smaller than it was in the complete-darkness condition. The horizon-and-stars condition simulated a natural outdoor setting; the silhouette of a city and of the skyline (horizon) and a starry sky were seen in the dark. The type of visual cues they provided were different from those in the lighted-room condition; the skyline and stars in the horizon-and-stars condition appeared to be far away, whereas the objects seen in the lighted-room condition appeared to be relatively nearby. Thus, in the horizon-and-stars condition, both visual and oculomotor cues might be available and might have played some role in inducing the illusion. But the smallness of the illusion relative to that in the complete-darkness condition suggests that the effects of visual and oculomotor cues might weaken each other to some degree rather than operating additively. As suggested above in connection with the absence of the illusion in the lighted-room condition, some visual cues provided precise information about size and distance, and therefore might occasionally weaken the illusion.

The presence of the illusion in the complete-darkness condition disagrees with Kaufman and Rock's (1962) inability to obtain the illusion. How did this discrepancy take place? One possibility is that of stray light. In their interpretation of Leibowitz and Hartman's (1959) and Schur's (1925) results, Kaufman and Rock (1962) proposed the possibility of stray light, but did not show that the illusion arises in a similar context when stray light is present. And, as shown in the results of the lighted- room condition in the present study, the illusion does not always occur when there are visual cues to size and distance perception. Kaufman and Rock's (1962) result of no illusion in complete darkness parallels the absence of the illusion in the lighted-room condition. The interpretation that Kaufman and Rock gave to Leibowitz and Hartman's and Schur's experiments may in fact, then, apply to their own experiments. That is, the visibility of objects in the room due to stray light might have prevented or weakened the illusion. Another possibility is that of the variability of the resting position of vergence among the subjects. Heuer and Owens (1989) reported that the effect of gaze direction on the resting position of vergence was variable among the subjects: Some subjects showed a small effect, while others showed a marked one. Thus, the individual difference in the effect of gaze direction on the resting position of vergence might have obscured the illusion.

Eye-and-head position affected the magnitude of the illusion. The obtained ratios were larger in the eyes-elevated condition than in the eyes-level condition. The size ratios of the eyes-elevated condition to the eyes-level condition were $1.33 / 1.26,1.08 / 1.00$, and 1.26/1.16-that is, 1.06 , 1.08 , and 1.10 for the complete-darkness, lighted-room, and horizon-and-stars conditions, respectively. These results agree with those obtained in Hermans's (1954) experiment. This effect is consistent also with the results obtained by Heuer and Owens (1989), showing that the effect of gaze direction on the resting position of vergence was larger in the eye-inclination condition than in the headinclination condition. Evidently, eye elevation affected the magnitude of the illusion, although Kaufman and Rock suggested that its effect on the magnitude of the illusion was negligible (Kaufman \& Rock, 1962; Rock \& Kaufman, 1962). But eye elevation can only partly explain the illusion. The usually observed moon illusion is thought to be a combined illusion: the illusion where the horizon moon appears larger and the illusion where the zenith moon appears smaller. In the present experiment, eye position was varied only when the subjects viewed the overhead stimulus. Thus, it can be said that eye elevation, one of the oculomotor cues, contributed to the illusion where the zenith moon appeared smaller. Holway and Boring were correct in this respect, although they were wrong in attributing a main cause of the illusion to eye elevation (Holway \& Boring, 1940a, 1940b).

Since publication of Kaufman and Rock's experiments (Kaufman \& Rock, 1962; Rock \& Kaufman, 1962), sizedistance invariance has been thought to be involved in the moon illusion. Recently, through ingenious experiments, Enright (1989b) showed that size-distance invariance cannot be applied to the moon illusion. Even though visible terrain may contribute to the illusion to some degree, the information of the oculomotor system may play a great role in the illusion, as proposed by Enright (1989a), Leibowitz and Owens (1989), and Roscoe (1989). The 
results of the complete-darkness condition in the present experiment also support this idea.

\section{REFERENCES}

ENRIGHT, J. T. (1989a). The eye, the brain, and the size of the moon: Toward a unified oculomotor hypothesis for the moon illusion. In $\mathrm{M}$. Hershenson (Ed.), The moon illusion (pp. 59-121). Hillsdale, $\mathrm{NJ}$ : Erlbaum.

ENRIGHT, J. T. (1989b). Manipulating stereopsis and vergence in an outdoor setting: Moon, sky and horizon. Vision Research, 29, 1815-1824.

Hermans, T. G. (1954). The relation of convergence and elevation changes to judgments of size. Joumal of Experimental Psychology, 48, 204-208.

Heuer, H., OWens, D. A. (1989). Vertical gaze direction and the resting posture of the eyes. Perception, 18, 363-377.

Holway, A. H., \& Boring, E. G. (1940a). The apparent size of the moon as a function of the angle of regard: Further experiments. American Journal of Psychology, 53, 537-553.

Holway, A. H., Boring, E. G. (1940b). The moon illusion and the angle of regard. American Journal of Psychology, 53, $109-116$.

IA vecChia, J. H., IAvecchia, H. P., Roscoe, S. N. (1983). The moon illusion revisited. Aviation, Space, \& Environmental Medicine, $54,39-46$.

KaUfman, L. \& Rock, I. (1962). The moon illusion, I. Science, 136, 953-962.
Kaufman, L., Rock, I. (1989). The moon illusion thirty years later. In M. Hershenson (Ed.), The moon illusion (pp. 193-234). Hillsdale, NJ: Erlbaum.

Leibowitz, H. W., Hartman, T. (1959). Magnitude of the moon illusion as a function of the age of the observer. Science, 130, 569-570.

LEIBowITZ, H. W., \& OWENS, D. A. (1989). Multiple mechanisms of the moon illusion and size perception. In M. Hershenson (Ed.), The moon illusion (pp. 281-286). Hillsdale, NJ: Erlbaum.

Rock, I., \& Kaufman, L. (1962). The moon illusion, II. Science, 136, 1023-1031.

Roscoe, S. N. (1989). The zoom-lens hypothesis. In M. Hershenson (Ed.), The moon illusion (pp. 31-57). Hillsdale, NJ: Erlbaum.

Schur, E. (1925). Mondtäuchung and Sehgrössenkonstant. Psychologische Forschung, 7, 44-80.

Stroobant, P. (1884). Sur l'agrandissement apparent des constellations, du soleil et de la lune à l'horizon. Bulletin de l'Académie Royale de Belgique (3rd series), 8, 719-731.

ThоR, D. H., \&OOD, R. J. (1966, May). A vestibular hypothesis for the moon illusion. Paper presented at the Annual Meeting of the Midwestern Psychological Association, Chicago.

ZoTH, O. (1899). Über den Einfluss der Blickrichtung auf die scheinbare Grösse der Gestirne und die scheinbare Form des Himmelsgewölbes. Pflügers Archiv fur die Gesamte Physiologie, 78, 363-401.

(Manuscript received August 8, 1990;

revision accepted for publication November 15, 1990.)

\title{
Announcement
}

\author{
Fifth Toledo Symposium on Advances in Biobehavioral Research \\ Mechanisms and Models of Interhemispheric Communication \\ University of Toledo, Driscoll Center \\ April 12-13, 1991
}

This symposium will present advances in theory, models, techniques, and research which characterize the nature of interhemispheric communication. The participants will be: M. Banich, D. Boles, C. Chiarello, S. Christman, R. Gur, C. Hamilton, C. Hardyck, L. Harris, J. Hellige, S. Levine, J. Liederman, L. Robertson, J. Sergent, S. Witleson, and E. Zaibel.

For further information, call F. Kitterle at 419-537-2722 or on E-mail FAC0532@UOFT01. 